\title{
¿QUÉ VEINTE AÑOS NO ES NADA...? UNA MIRADA A LA DESIGUALDAD DE INGRESOS A PARTIR DE LAS ENCUESTAS CASEN
}

\section{Harald Beyer}

Desde 1990 se han realizado nueve encuestas Casen. Ellas recogen una importante información socioeconómica que permite ilustrar el diseño de la política social, pero también comprender la evolución de la pobreza y la desigualdad de ingresos en Chile. La primera variable muestra una tendencia declinante, más allá de la experiencia de 2009 que estuvo influida por el alza en el precio de los alimentos y las características particulares de la metodología que tradicionalmente se ha empleado para medir la pobreza en nuestro país. Este estudio aborda la segunda de estas dimensiones: la desigualdad de ingresos. A diferencia de lo que ha sucedido con la pobreza ésta parece no ceder y mantiene una estabilidad en niveles altos. Además, la última medición reveló un importante aumento, respecto de encuestas anteriores, en la razón de los ingresos autónomos del décimo y del primer decil de ingresos. Sin embargo, ese aumento en la brecha estuvo muy ligado a la crisis económica que vivió el país, al igual que el resto del mundo, durante 2009.

Harald Beyer. Ingeniero Comercial, Universidad de Chile. Ph. D. en Economía, Universidad de California, Los Angeles. Investigador y coordinador académico del Centro de Estudios Públicos. 
Una mirada más amplia revela que, a pesar de que los cambios son aún modestos y se avanza gradualmente, la dirección que sugieren las cifras de desigualdad reportadas por las encuestas Casen es positiva. Así, un análisis cuidadoso de los datos indica un progreso en este ámbito que la crisis no parece haber detenido, aunque indudablemente golpeó a través del impacto sobre el empleo y, por esta vía, en los ingresos autónomos de los hogares. El análisis que aquí se presenta y que pone atención en la evolución de los ingresos autónomos, los ingresos del trabajo y el efecto del aumento en la escolaridad de la población permite augurar que en el futuro la desigualdad podría seguir reduciéndose como ocurrió entre 2000 y 2006 una vez que queden atrás los inevitables efectos negativos de la crisis económica reciente. La debilidad en este cuadro positivo es el empleo, que no sólo es reducido cuando se lo compara con otros países sino que es especialmente así entre los grupos menos calificados. Los gobiernos interesados en la equidad deberían poner cada vez más atención a su evolución, porque es una herramienta muy poderosa para avanzar en ese propósito.

Palabras clave: distribución del ingreso; pobreza; escolaridad; empleo.

Recibido y aceptado: diciembre 2010.

\section{Introducción}

$\mathrm{E}_{\mathrm{s}}$ sabido que Chile tiene un alto nivel de desigualdad. Ésta es una particularidad que comparte con otros países de la región latinoamericana. En efecto, nuestro país se ubica aproximadamente en la zona media en materia de desigualdad entre los países de la región. Ahora bien, ésta es a su vez de las más desiguales del planeta, sólo superada por el África Subsahariano. A pesar que en los últimos 25 años Chile ha exhibido un buen crecimiento económico que lo ha acercado a los países en desarrollo, a diferencia de lo que ocurrió en buena parte del siglo XX cuando más bien se alejaba de ellos, la situación de desigualdad del país parece persistir. No parece estar importando demasiado que el país haya invertido en la escolaridad de su población, que el gasto social haya crecido y que éste se gaste en proporciones importantes en los menos aventajados. Por cierto, hay programas inefectivos, pero ello no significa que la orientación general de la política social sea equivo- 
cada $^{1}$. A pesar de ello los resultados en términos de modificación de la desigualdad son en apariencia insuficientes. Ello genera, en algunos sectores, un cuestionamiento al así llamado "modelo de desarrollo" que el país ha venido consolidando en los últimos lustros y, en otros, un llamado a elevar la carga tributaria para profundizar programas vigentes o crear otros que busquen más activamente promover una mayor igualdad de resultados. De algún modo ambas miradas se entrelazan y se pretenden esfuerzos adicionales que impacten la desigualdad de ingresos.

Sin embargo, no hay claridad respecto de cómo esos esfuerzos podrán conseguir los propósitos enunciados. Muchas veces, los argumentos se escudan en la experiencia comparada, particularmente en el hecho de que una gran mayoría de países de igual o superior ingreso per cápita exhiben tanto un mayor gasto público como una menor desigualdad (hay, por cierto, excepciones a esta regla). Con todo, no es evidente, que la gran clave para reducir las desigualdades de esos países esté en su gasto público. Es cierto que los indicadores tradicionales de desigualdad mejoran una vez que se incorporan las transferencias netas a los hogares (subsidios menos impuestos), pero el mejoramiento es a partir de valores que son bastante menores que los nacionales. Precisamente, que el punto de partida sea bastante más igualitario es lo que hace que esas transferencias netas reduzcan aún más las desigualdades ${ }^{2}$. En ese sentido si bien el debate respecto de cuánto transferir y cómo hacerlo no debe escabullirse, no puede olvidarse que la distribución de ingresos autónomos en Chile, es decir aquellos generados por los propios hogares sin asistencia gubernamental, es muy desigual respecto de países de mayor gasto público y nivel de ingresos.

Es indispensable, entonces, poner atención a la evolución de los ingresos autónomos de modo independiente al debate respecto del nivel apropiado de gasto público. En el caso de Chile, además, es importante reconocer que una parte importante de los bienes públicos y del gasto que en otras latitudes es de cargo de los gobiernos, en nuestro país es financiado privadamente. La mirada a esos ingresos autónomos y a su distribución es, en una primera aproximación, negativa. En los últimos años no parece haberse modificado mayormente el coeficiente Gini ni (2004).

${ }^{1}$ Una revisión de la política social reciente se puede encontrar en Arellano

2 Sobre las desigualdades antes y después de transferencias netas para varios países desarrollados puede consultarse Kenworthy (2008). 
las brechas de ingreso entre los hogares más y menos acomodados. Por cierto, ello puede ser el resultado de que los indicadores analizados son demasiado generales como para apreciar los cambios que puedan estar ocurriendo. También puede ser que su nivel de agregación impide ver la dinámica subyacente y que ésta vaya en la dirección esperada. En particular, que esté ocurriendo un proceso más profundo de movilidad social del que se advierte a simple vista o que todavía exista un rezago consecuencia de una historia de insuficiente e incorrecta inversión en los grupos sociales más desaventajados ${ }^{3}$.

Quizás también esta falta de avances pueda obedecer a que se mide con una vara muy exigente los cambios que han ocurrido en los indicadores tradicionales. No cabe duda que mover la distribución del ingreso de un país no es un hecho que pueda ocurrir en plazos breves, más todavía cuando están ocurriendo fenómenos de diversa índole que en el corto plazo pueden jugar en contra de este propósito y que son propios de una economía que aún no se consolida. Por ejemplo, la expansión de la tasa de participación femenina ha ocurrido muy gradualmente y se ha producido con más fuerza entre las mujeres de más alto nivel escolar. Así, entre 1990 y 2009 la tasa de participación de la mujer de doce años de escolaridad y más subió de 49 a 56 por ciento mientras que la de siete años y menos se mantuvo constante en 22 por ciento. La participación de la mujer con escolaridad de entre 8 y 11 años subió en el mismo período desde un 26 a un 32 por ciento. Si se considera que la escolaridad de las parejas está altamente correlacionada en Chile, el efecto sobre la desigualdad de ingresos de esta dispar evolución en las tasas femeninas de participación es evidente.

Aunque todo esto es cierto, es posible detenerse en los indicadores más habituales y distinguir algunos elementos que hacen pensar que si bien la desigualdad no va a reducirse de manera abrupta, las posibilidades de que estemos frente a un proceso gradual de avances en esta dimensión no se pueden desechar del todo. Los antecedentes disponibles sugieren que estos avances son consistentes y que ellos no se diluyeron con la crisis económica de fines de 2008 y del primer semestre de 2009. Destacan al respecto los cambios en la forma en que se

${ }^{3}$ Sapelli (2009) hace un buen análisis respecto de estas materias y observa mirando cambios en la desigualdad entre generaciones y recogiendo la información disponible sobre movilidad social que los indicadores tradicionales no permiten apreciar los enormes cambios que se avecinan en reducción de desigualdades y mayor movilidad social. 
estructura la distribución del ingreso autónomo per cápita, la reducción en las diferencias salariales y el cierre de las brechas de escolaridad. Por supuesto, estos cambios han sido acompañados por otros que exhiben un comportamiento que es contradictorio con el propósito de reducir la desigualdad. En particular, la evolución del empleo sugiere que se ha ido reduciendo el espacio para los trabajadores menos calificados. Este estudio repasa en las secciones siguientes estos aspectos. El panorama que emerge de esta labor es que la estabilidad que se observa en la distribución del ingreso es más aparente que real y que hay cambios que auguran que en el futuro se podría observar una reducción no despreciable en la desigualdad de ingresos.

\section{Los indicadores agregados}

La forma tradicional de evaluar la evolución de la desigualdad es a través de las razones de ingresos entre deciles o entre quintiles y el coeficiente Gini ${ }^{4}$. Los informes oficiales sobre desigualdad típicamente reportan estos indicadores. El Cuadro $\mathrm{N}^{\circ} 1$ reproduce algunos de los antecedentes divulgados en la última medición de la encuesta Casen correspondiente a 2009 para los ingresos autónomos y monetarios. Es importante aclarar que para ordenar a los hogares se calcula el ingreso autónomo per cápita de cada uno de ellos. Se ordena a los hogares de acuerdo a este indicador de menor a mayor y se dividen en diez grupos iguales. Ese proceso determina cada decil. Así, la razón 10/10 divide la proporción del ingreso autónomo total que recibe el 10 por ciento de mayores ingresos y el 10 por ciento de menores ingresos. Como en Chile, producto de la elevada desigualdad, la proporción de ese ingreso que captura el décimo decil es elevada, ese indicador es muy sensible a pequeños cambios en esas proporciones. Ello explica el fuerte aumento en la razón 10/10 entre 2006 y 2009. El coeficiente Gini muestra un cambio menor y resulta evidente que en 2009 la desigualdad sigue siendo menor que en 2000 y que los años previos. Por ello es importante mirar en conjunto todos los indicadores antes de extraer conclusiones muy precipitadas.

${ }^{4}$ Hay que recordar que este coeficiente puede tomar valores entre 0 y 100 (o entre 0 y 1 ), siendo este último el valor que se le asigna al coeficiente en caso de desigualdad absoluta (todo el ingreso de un país se concentra en una sola mano). Si toma el valor cero significa que todos los hogares o individuos acceden a igual proporción del ingreso nacional y, por tanto, hay igualdad total. 
CUADRO N ${ }^{\circ}$ 1: INDICADORES DE DESIGUALDAD PARA INGRESOS AUTÓNOMOS Y MONETARIOS

(Hogares ordenados por ingreso autónomo per cápita)

\begin{tabular}{lccccc}
\hline & 1990 & 1996 & 2000 & 2006 & 2009 \\
\hline Autónomos & & & & & \\
Razón 10/10 & 30,5 & 33,0 & 34,2 & 31,3 & 46,0 \\
Gini & 0,57 & 0,57 & 0,58 & 0,54 & 0,55 \\
Monetarios & & & & & \\
Razón 10/10 & 27,1 & 25,7 & 29,5 & 23,9 & 25,9 \\
Gini & 0,56 & 0,56 & 0,58 & 0,53 & 0,53 \\
\hline
\end{tabular}

Fuente: Mideplan.

En este caso particular parece razonable concluir que ha habido un cambio no despreciable en la desigualdad a partir de 2000 y que la crisis de 2009, a pesar de su profundidad, no lo revirtió. Para sostener este punto es indispensable dar cuenta de por qué aumentó la razón 10/10 para los ingresos autónomos. El Gráfico $\mathrm{N}^{\mathrm{o}} 1$ es ilustrativo al respecto.

\section{GRÁFICO No ${ }^{\circ}$ 1: VARIACIÓN PORCENTUAL EN INGRESOS AUTÓNOMOS PER CÁPITA ENTRE 2006 Y $2009^{5}$ \\ (Por centil de ingreso per cápita de los hogares)}

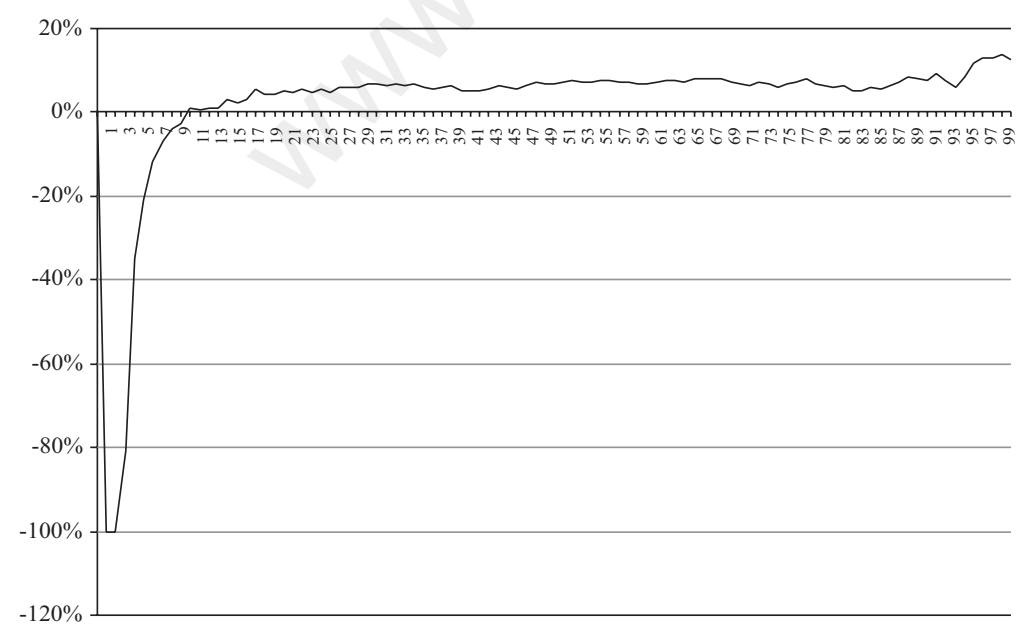

Fuente: Elaboración propia a partir de encuestas Casen.

${ }^{5}$ Respecto del gráfico es importante tener en cuenta que en ambos años el centil de menores ingresos era igual a cero. Para estos casos la variación porcentual no está definida y, por ello, el Gráfico tiene en su extremo izquierdo esa forma tan particular. 
Es evidente que hubo una caída muy importante en los ingresos de algunos hogares, al grado de que poco más de un tres por ciento de los hogares reporta ingresos autónomos iguales a cero (típicamente en las encuestas Casen sólo un uno por ciento de los hogares reporta ingresos iguales a cero). Los siguientes centiles también reportan caídas en sus ingresos reales y sólo a partir del percentil 10 comienzan a manifestarse variaciones positivas en el ingreso autónomo per cápita de los hogares. Si se piensa que la principal fuente de ingresos autónomos es el trabajo, no cabe duda que este fenómeno estuvo relacionado con la pérdida de empleo o la imposibilidad de mantener un empleo durante el año 2009. Por cierto estos números también revelan las limitaciones de una encuesta que sólo realiza una medición puntual. Es poco probable que esa caída de ingresos autónomos haya sido permanente durante, por ejemplo, todo el año 2009.

Ahora bien, el Cuadro $\mathrm{N}^{\circ} 1$ también muestra que el cambio en la razón 10/10 para los ingresos monetarios sufre variaciones bastante menores que las observadas para los ingresos autónomos, particularmente entre 2006 y 2009. Se revela así el efecto moderador que las transferencias en dinero tienen en períodos de crisis. Por cierto, estas transferencias no son muy significativas, algo que se puede ver en el hecho de que los coeficientes Gini para ingresos autónomos y monetarios son prácticamente equivalentes ${ }^{6}$. Sin embargo, para los grupos de ingresos reducidos no dejan de ser relevantes. Ello se puede ver más claramente en el Cuadro $\mathrm{N}^{\mathrm{o}} 2$ que presenta las razones 10/10 en 2006 y 2009, pero ahora ordenando los hogares de dos formas alternativas: de acuerdo al ingreso monetario per cápita de los hogares, de modo de reconocer las transferencias en dinero, y de acuerdo al ingreso per cápita total, que suma al factor anterior el arriendo imputado por la propiedad de vivienda. Este último factor es importante de considerar porque se asemeja al concepto de ingreso permanente, toda vez que la vivienda propia genera un flujo de servicios que tiene un valor económico que no se puede desconocer.

Es claro que las razones 10/10 tienden a disminuir, porque proporcionalmente los subsidios en dinero y la imputación por vivienda representan una parte más importante de los ingresos autónomos para los hogares de menores ingresos. Además, al ordenarse por ingreso mone-

${ }^{6}$ Esto también ocurriría si los subsidios monetarios se entregaran más o menos en proporción a la distribución de los ingresos autónomos, pero sabemos que ellos están relativamente bien focalizados. 
CUADRO Nº 2: $\quad$ RAZÓN 10/10 PARA INGRESOS MONETARIOS Y TOTALES EN 2006 Y 2009

(Hogares ordenados por ingresos monetarios y totales per cápita)

\begin{tabular}{lcc}
\hline & 2006 & 2009 \\
\hline & 22,2 & 24,5 \\
Ingresos monetarios & 18,9 & 20,6 \\
Ingresos totales & & \\
\hline
\end{tabular}

Fuente: Elaboración propia a partir de encuestas Casen.

tario y total per cápita se recogen de mejor forma las interacciones entre los ingresos autónomos de los hogares, las características propias de la política social y la situación de vivienda. Por cierto, a partir de estos antecedentes agregados no es posible explorar las perspectivas futuras sobre la desigualdad de ingresos. Las siguientes secciones indagan más sobre estos asuntos.

\section{Una mirada más integral a la distribución de ingresos}

La persistencia de la desigualdad no es algo que debería sorprender, toda vez que la inversión en áreas relevantes para revertir esta situación como es, por ejemplo, la masificación de la educación tomó mucho tiempo ${ }^{7}$. El Gráfico $\mathrm{N}^{0} 2$, elaborado para distintas cohortes de edad sobre la base de la encuesta Casen 2009, es un modo didáctico de presentar ese atraso. La escolaridad de las generaciones mayores no sólo era, en promedio, reducida sino que exhibía una alta dispersión medida por la varianza. Es decir, un pequeño grupo tenía una alta escolaridad y una proporción relativamente grande exhibía bajos niveles de escolaridad. Se constata que sólo en las generaciones más jóvenes se han alcanzado

${ }^{7}$ No es fácil saber exactamente cuánto era la desigualdad en Chile en el pasado lejano, pero de acuerdo a Williamson (2009) hacia 1861 el coeficiente Gini alcanzaba un valor de 63,7. El estudio de Williamson sugiere, además, que los países latinoamericanos no habrían estado entre los más desiguales con anterioridad a la última parte del siglo XIX. Incluso es posible que en los siglos anteriores hayan sido más igualitarios que los europeos. El gran distanciamiento se habría producido, entonces, en los últimos 150 años y particularmente durante el siglo XX. En su opinión la evidencia, aunque reconoce que es dispersa, contrasta con la convención más aceptada de que América Latina siempre fue desigual. 
GRÁFICO No 2: PROMEDIO Y VARIANZA DE ESCOLARIDAD PARA DISTINTAS COHORTES DE EDAD



Fuente: Elaboración propia sobre la base de Casen 2009.

niveles razonables de escolaridad. En estos grupos se observa no sólo un aumento en la escolaridad sino que menores brechas al interior de ellos que explican la menor dispersión graficada en esta figura.

Una forma alternativa de visualizar los cambios que han ocurrido en materia de escolaridad es revisar el Cuadro $\mathrm{N}^{\circ} 3$ que compara la distribución de escolaridad de la población entre 25 y 34 años (es decir los nacidos entre 1975 y 1984) y la de 55 a 64 años (es decir los nacidos entre 1945 y 1954).

CUADRO N ${ }^{\circ}$ 3: DISTRIBUCIÓN DE ESCOLARIDAD PARA DOS COHORTES DE EDAD

\begin{tabular}{lcc}
\hline & $25-34$ años & $55-64$ años \\
\hline $0-8$ & $15 \%$ & $50 \%$ \\
$9-12$ y más & $49 \%$ & $33 \%$ \\
& $36 \%$ & $17 \%$ \\
\hline
\end{tabular}

Fuente: Elaboración propia sobre la base de Casen 2009. 
Es evidente que la escolaridad de la población ha cambiado de modo significativo en el lapso de una generación (30 años). Estos cambios y aumentos en la escolaridad comienzan a manifestarse cada vez con más intensidad en la fuerza de trabajo. El Cuadro $\mathrm{N}^{\circ} 4$ muestra la distribución por escolaridad de la fuerza de trabajo en 1990 y 2009.

CUADRO N ${ }^{\circ}$ 4: DISTRIBUCIÓN POR ESCOLARIDAD DE LA FUERZA DE TRABAJO

\begin{tabular}{lll}
\hline & 1990 & 2009 \\
\hline $0-8$ & & \\
$9-12$ & $40 \%$ & $24 \%$ \\
13 y más & $38 \%$ & $48 \%$ \\
& $22 \%$ & $28 \%$ \\
\hline
\end{tabular}

Fuente: Elaboración propia sobre la base de encuestas Casen 1990 y 2009.

Por cierto, las brechas de escolaridad seguirán reduciéndose y se observarán cambios adicionales en la distribución por años de educación. Si se invierte apropiadamente ${ }^{8}$, este cierre es natural toda vez que la escolaridad formal tiene techo y que sus aumentos entre la población más joven elevan gradualmente los mínimos de escolaridad en la fuerza de trabajo. Como en ésta aún permanece un grupo de personas con baja escolaridad el efecto sobre la desigualdad de ingresos es incierto en una primera etapa. La razón de ello es que al interior de los grupos más jóvenes el cierre de brechas en educación efectivamente puede reducir las diferencias de ingreso, dado que ambas variables están positivamente correlacionadas. Sin embargo, el aumento de la escolaridad de las generaciones más jóvenes puede aumentar las diferencias de ingreso con las mayores. Así, el efecto sobre la desigualdad de ingresos medida, en este caso, por la varianza de estos es incierta. Por supuesto, son muchos otros factores que están influyendo en la evolución de los ingresos de hogares y personas. Algunos de ellos están influidos directa o indirectamente por la educación como la caída en la tasa de fecundidad o la mayor participación de la mujer en la fuerza de trabajo. Otros no están re-

${ }^{8}$ Este aspecto es importante tenerlo en mente, porque a menudo se da por sentado que se invertirá apropiadamente, pero no siempre ocurre así como sugiere la propia experiencia chilena durante una buena parte de su historia como nación independiente. 
lacionados con aspectos educacionales como son los ciclos económicos, los cambios tecnológicos ${ }^{9}$, la importancia, financiamiento, efectividad y foco del gasto público, las características de los flujos internacionales de bienes y servicios, las modificaciones en precios relativos relevantes para la economía nacional o la transformación de regulaciones importantes en los mercados de bienes y factores productivos nacionales ${ }^{10}$. Por cierto, ese listado no pretende ser exhaustivo y tampoco establecer el peso relativo de cada uno de sus componentes, si alguno, en el aporte a la desigualdad que se observa en el país.

La interacción de todos estos elementos hace difícil distinguir entre aspectos permanentes y circunstanciales en la desigualdad de ingresos. Pero ello no significa que los primeros no estén presentes. Así, por ejemplo, si contrastamos el coeficiente de Gini hacia 1861 y el actual, observamos una reducción relevante en la desigualdad aunque no podamos identificar con exactitud el momento en que se produjo ese quiebre y qué lo causó. Probablemente ocurrió con gradualidad y como consecuencia de un conjunto de elementos no todos observados en su momento, reflejo de que en esta dimensión los cambios ocurren sin que sean notados con precisión. Ahora que se reúne información sobre ingresos con más periodicidad y que ésta suele ser de mejor calidad quizás los cambios siguientes puedan comenzar a detectarse con anticipación. Al respecto, el Gráfico $\mathrm{N}^{\circ} 3$ puede ser ilustrativo. En él se muestra la distribución de los ingresos autónomos per cápita de los hogares para distintos años en las últimas dos décadas.

Una lectura cuidadosa del Gráfico $\mathrm{N}^{\mathrm{o}} 3$ permite apreciar que entre 1990 y 2000 la forma de la curva que representa el ingreso autónomo per cápita del hogar, ordenado por percentil de la distribución, no sufre mayores modificaciones. Es un desplazamiento básicamente paralelo de la curva sugiriendo que todos los hogares modificaron sus ingresos más o menos en la misma proporción, pero que la brecha entre ellos se mantuvo relativamente parecida. Entre 2000 y 2006, en cambio, el desplazamiento no es paralelo. Es más pronunciado en la parte inferior de la distribución sugiriendo una compresión en las brechas de ingreso. De hecho el coeficiente de Gini cayó desde 0,58 a 0,54 entre 2000 y

${ }^{9}$ Aunque en este caso se podría argumentar que está influido por cambios educacionales en otros lugares del globo.

${ }^{10}$ Sobre esto puede verse Beyer (2005) y la literatura ahí citada. 
GRÁFICO Nº 3: DISTRIBUCIÓN DEL INGRESO AUTÓNOMO PER CÁPITA DEL HOGAR POR CENTIL

(Cifras corresponden al logaritmo natural del ingreso en pesos de noviembre de 2009)

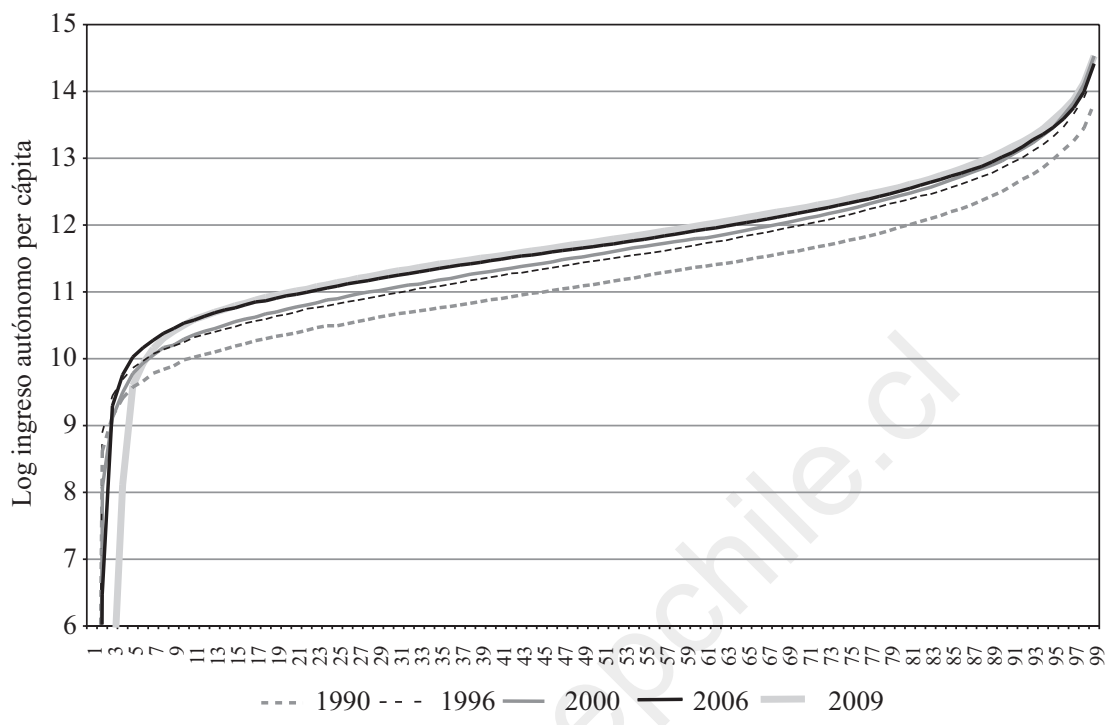

Percentil del ingreso autónomo per cápita del hogar

2006 siendo ese último año el valor más bajo desde $1990^{11}$. Un aspecto interesante es que en 2009, a pesar de la profunda crisis económica previa al momento en que se tomó la encuesta Casen la forma de la curva no sufrió un cambio estructural. Es posible apreciar que no cambió mayormente respecto de la observada en 2006 excepto en la parte inferior de la distribución donde la curva se desploma. Ello es consecuencia, como veremos más adelante, del importante retroceso que se observó en el empleo de algunos hogares ${ }^{12}$.

${ }^{11}$ Larrañaga y Herrera (2008) ya hicieron notar este fenómeno, atribuyéndolo a la caída en la tasa de natalidad en los grupos de menores ingresos que contribuye a elevar el ingreso per cápita de los hogares. El fenómeno se extendía a otras mediciones de ingreso como consecuencia de la reducción de desigualdad entre trabajadores de distintos niveles de escolaridad y una menor dispersión de salarios al interior de cada categoría educacional.

${ }^{12}$ Una aclaración técnica es necesaria en este punto. Hay que recordar que el Gráfico $\mathrm{N}^{\mathrm{o}} 3$ es una transformación logarítmica de los ingresos autónomos per cápita de los hogares. Típicamente en la Casen el primer centil aparece con ingresos autónomos iguales a cero. Para ese valor el logaritmo no está definido, de ahí que las curvas del Gráfico $\mathrm{N}^{\circ} 3$ se quiebren hacia el comienzo de la distribución. En la encuesta Casen de 2009 el tres por ciento de los hogares de menores rentas reporta ingresos autónomos iguales a cero. 
Esa compresión de las brechas que ocurre como una respuesta a un aumento relativo en el ingreso per cápita de los hogares que están en la parte inferior de la distribución va de la mano con la evolución de los ingresos del trabajo que se presenta en el Cuadro $\mathrm{N}^{0} 5$. Es evidente que las diferencias en el logaritmo del ingreso laboral por hora han disminuido. Los cambios son importantes y se explican fundamentalmente por lo ocurrido en la parte inferior de la distribución de ingresos, donde se observa un mayor grado de compresión. Se aprecia, además, que estos cambios ocurrieron con más fuerza entre 2000 y 2006 y que en 2009, a pesar de la crisis, la nueva estructura de ingresos de 2006 no sufrió mayores variaciones.

CUADRO N ${ }^{\circ}$ 5: DIFERENCIAS EN EL LOGARITMO NATURAL DE LOS INGRESOS DEL TRABAJO POR HORA

(Diferencias para los percentiles 90, 50 y 10)

\begin{tabular}{llllll}
\hline & 1990 & 1996 & 2000 & 2006 & 2009 \\
\hline P90-P10 & 2,15 & 2,28 & 2,11 & 1,99 & 2,01 \\
P90-P50 & 1,33 & 1,38 & 1,33 & 1,30 & 1,31 \\
P50-10 & 0,82 & 0,90 & 0,78 & 0,69 & 0,70 \\
\hline
\end{tabular}

Fuente: Elaboración propia sobre la base de encuestas Casen.

Esta reducción en las brechas, sobre todo en la parte inferior de la distribución de los ingresos del trabajo, puede haber sido influida por muchos factores, pero no cabe duda que los aumentos en la escolaridad en la fuerza de trabajo, reportados previamente, pueden haber influido en esta realidad. Quizás ello se aprecie mejor si observamos con más precisión cómo cambió la distribución de escolaridad de la fuerza de trabajo entre 1990 y 2009. El Gráfico N 4 presenta la distribución de escolaridad de la fuerza de trabajo por veintil (esto es, se divide la fuerza de trabajo en veinte grupos iguales y se observa cuál es la escolaridad que divide a cada uno de los grupos) en 1990 y 2009. En la parte inferior de la distribución se observa que la escolaridad ha crecido para cada percentil reportado entre dos y tres años. En particular, la mediana de la escolaridad de la fuerza de trabajo aumentó en dos años entre 1990 y 2009 siendo ahora equivalente a educación media completa. 
GRÁFICO No 4: DISTRIBUCIÓN DE LA ESCOLARIDAD DE LA FUERZA DE TRABAJO

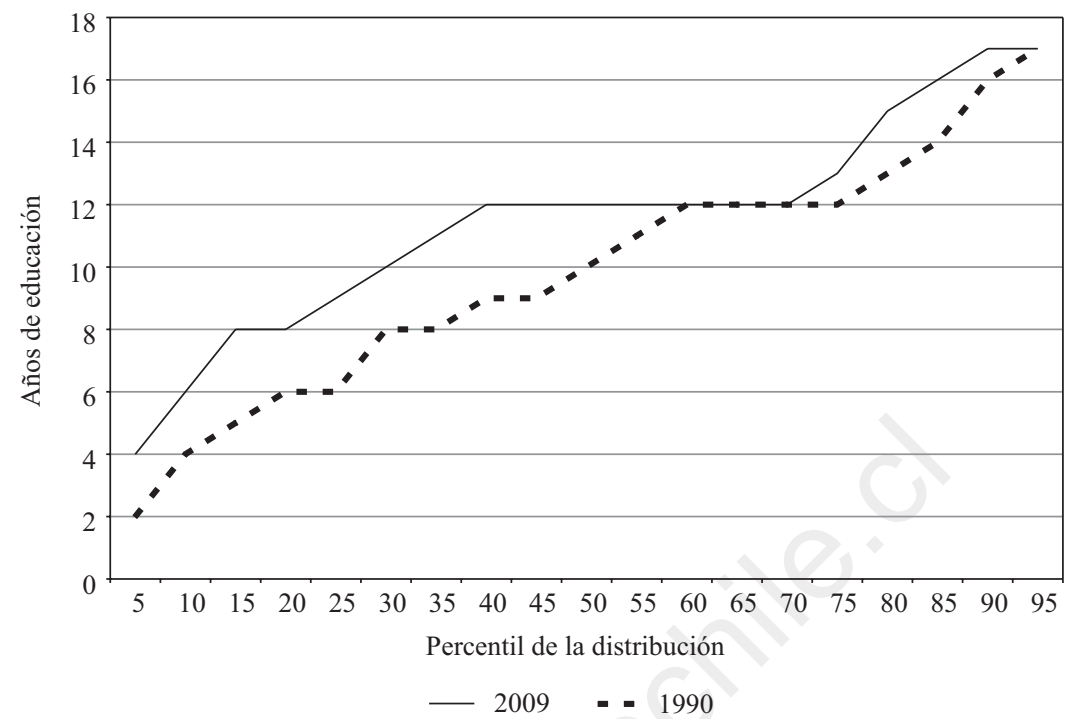

Fuente: Elaboración propia sobre la base de encuestas Casen 1990 y 2009.

Es evidente, además, que hay un aumento significativo de la fuerza de trabajo con doce años de educación. Ese aumento en la oferta relativa de graduados de la educación media debe tenerse en mente al momento de evaluar otros fenómenos ocurridos en las dos últimas décadas. Es posible detectar en el gráfico que ha subido la escolaridad promedio de los que cursan educación superior (la curva de 1990 va por encima de la de 1990) y que si bien ha habido un aumento en la proporción de personas que cursan algún año de educación superior ese incremento ha sido relativamente modesto, sobre todo si se compara con los cambios en la tasa de personas que completaron la educación secundaria.

Sin perjuicio de los cambios positivos ocurridos en la parte inferior de la distribución de ingresos siguen llamando la atención las enormes brechas que se observan en su parte superior. Ellas no sólo son significativas sino que muy elevadas cuando se las compara con la realidad observada en otros países más igualitarios que el nuestro ${ }^{13}$. Es conocido

${ }^{13}$ Las diferencias en ingreso por hora para el P90 y el P50 son típicamente entre dos y tres veces más. En efecto, ellas fluctúan entre 0,38 para países como Noruega hasta 0,78 para países como Suiza (Blau y Kahn, 1996 y 2005). Si se piensa que en Chile este valor es 1,31 las enormes diferencias quedan en evidencia. 
el hecho de que ello está muy relacionado con el elevado premio a la educación superior en Chile, premio que ha subido fuertemente desde los 60 y que no muestra signos de debilitamiento ${ }^{14}$. Sobre ello trata la sección siguiente.

\section{EI peso de la educación}

En el modelo tradicional de Mincer (1974) se asume que el ingreso del trabajo (en particular el logaritmo natural de ese ingreso) es una función de la escolaridad. Así, la relación entre ambas variables se puede explicitar por medio de la siguiente ecuación.

$$
\operatorname{Ln} y=\alpha+\beta \cdot S+\mu,
$$

donde Ln y representa el logaritmo natural del ingreso del trabajo (o salario) por hora y $\mathrm{S}$ la escolaridad de la persona; $\mu$ es el error de esta ecuación. Indudablemente esta expresión se puede extender para incorporar otras variables relevantes como pueden ser la experiencia, el género del trabajador, la región en la que presta sus servicios, su estado civil, etc. En general, el modelo es bastante flexible para acomodar todas aquellas variables que se crea puedan contribuir a explicar los ingresos laborales de una persona. Una base de datos, como la Casen, que recoge información sobre los ingresos de las personas y sus distintas características permite estimar esta ecuación y obtener una relación entre las variables explicativas y el ingreso de las personas. Típicamente, en este tipo de estimaciones la escolaridad permite explicar hasta un 40 por ciento de la varianza en los ingresos. Probablemente ello subestima el impacto de la educación porque ella también actúa indirectamente afectando otros comportamientos de la población. Asimismo, los años de escolaridad es probablemente un indicador imperfecto de las habilidades que se adquieren a través de esta inversión.

Ahora bien, tal como está expresada esa ecuación ella supone que el retorno a la educación, esto es cuánto agrega al ingreso un año adicional de educación, es independiente del nivel educativo que se esté considerando. En particular, asume que el valor estimado de $\beta$ es constante para cada nivel educativo. Sin embargo, la particular estructura

\footnotetext{
${ }^{14}$ Sobre esto véase Beyer y Le Foulon (2002).
} 
de los ingresos del trabajo en Chile, en particular que ellos aumentan su valor a tasas crecientes cuando se analiza a personas con educación superior, hace difícil aceptar ese enfoque tradicional. Sin embargo, el enfoque se puede corregir sin mayores problemas para aceptar que el premio a la educación difiere según cuál sea el nivel educativo que se esté analizando. Para evaluar qué ha pasado con el retorno a la educación en distintos niveles educativos entre 1990 y 2009 se ha procedido a estimar la ecuación antes informada bajo el supuesto de que los retornos son diferentes en los primeros ocho años de escolaridad de los cuatro siguientes y éstos a su vez de los que se obtienen en la educación superior ${ }^{15}$. Las estimaciones se han hecho separadamente para asalariados y asalariadas y se ha controlado por experiencia aparente, zona de residencia y si la persona es jefe de hogar. El Gráfico $\mathrm{N}^{0} 5$ presenta los ingresos predichos asociados sólo a escolaridad de los asalariados y, por consiguiente, los retornos implícitos para los distintos niveles educativos. Lo hace para distintos años. El Gráfico $\mathrm{N}^{\mathrm{o}} 6$ realiza el mismo ejercicio, pero para las asalariadas.

Es evidente de ambos gráficos que hacia el final del período hay una reducción en el retorno a la educación básica (la pendiente de la curva para los primeros ocho años se torna más plana) tanto para hombres como mujeres. Hay, por consiguiente, una reducción en las diferencias salariales de estos grupos. Ello se explica en una parte importante por un mayor crecimiento del salario base en la economía, es decir aquel para personas sin educación. Ello puede ser el resultado de que las personas con baja escolaridad y sin muchas calificaciones están saliendo de la fuerza de trabajo. Algo de ello se verifica en la sección 5 y cabe preguntarse si sus sustentos fuera de la fuerza de trabajo son aceptables. Aunque es algo más errática la tendencia también es posible observar una leve disminución en la tasa de retorno de las personas que acceden a educación secundaria y que no persiguen estudios superiores. Ello es sobre todo evidente en los hombres, particularmente respecto de 1996. Esa situación no es tan clara en las mujeres, donde el retorno a la educación secundaria exhibe un patrón más errático, quizás como resultado de que su incorporación a la fuerza de trabajo es aún muy reducida comparada con la experiencia de otros países. En todo caso el retorno para la educación secundaria entre las mujeres es en 2009 más reducido que en 1990.

${ }^{15}$ Una explicación de cómo proceder para estos efectos se encuentra en Beyer (1999). 
GRÁFICO N ${ }^{\circ}$ 5: RETORNOS DE LA EDUCACIÓN PARA LOS ASALARIADOS EN DISTINTOS AÑOS

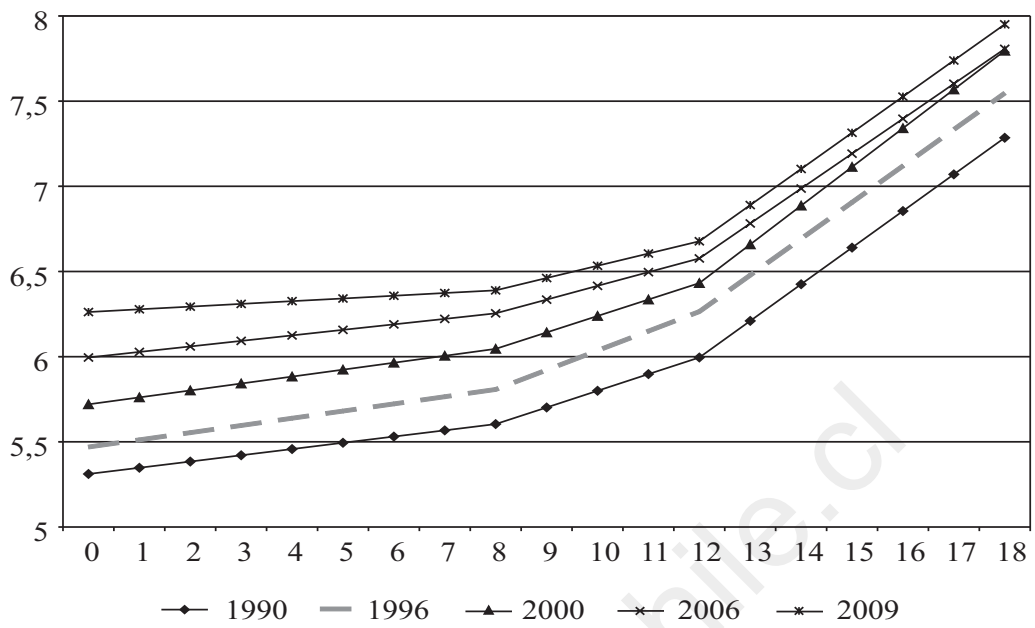

Fuente: Estimaciones obtenidas a partir de las encuestas Casen.

GRÁFICO N 6: RETORNOS DE LA EDUCACIÓN PARA LAS ASALARIADAS EN DISTINTOS AÑOS

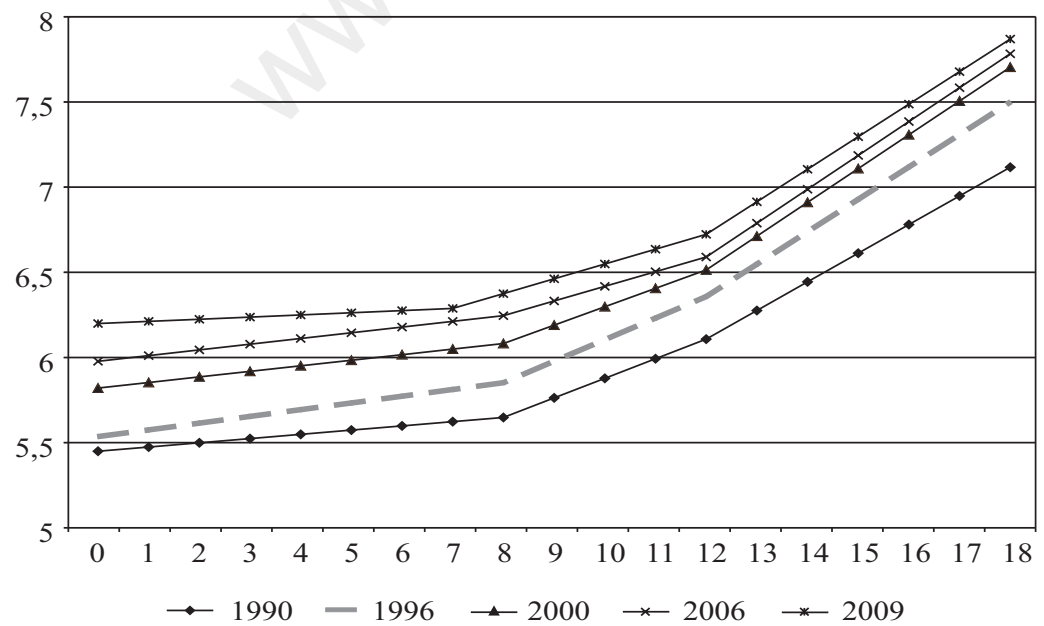

Fuente: Estimaciones obtenidas a partir de las encuestas Casen. 
Estos cambios en el retorno a la educación en básica y media parecen ser fundamentales a la hora de entender las menores diferencias en la parte inferior de la distribución de ingresos del trabajo, lo que está presionado por una mayor escolarización de la población y seguramente una autoselección de acuerdo a su calificación de las personas con muy reducido nivel de escolaridad. Los retornos para la educación superior, a pesar del aumento en la proporción de personas con trece años y más de escolaridad entre 1990 y 2009, parecen no ceder. Por cierto, el Cuadro $\mathrm{N}^{\circ} 4$ indicaba que la proporción de personas con trece años o más de escolaridad había aumentado sólo en seis puntos porcentuales: desde un 22 a un 28 por ciento. Este incremento es pequeño para una economía que desde 1990 ha tenido un crecimiento significativo y que, además, ha aumentado su nivel de apertura comercial, lo que aparentemente habría aumentado la demanda por capital humano por parte de las empresas ${ }^{16}$. En el caso de los hombres, la encuesta de 2006 sugería que podía haber un respiro y que finalmente el retorno a la educación comenzaba a ceder. Pero en la última encuesta esa sensación se diluyó y el retorno a la educación superior pasó a ser tan elevado como en las mediciones previas. En el caso de las mujeres el problema es aún más marcado. Se observa que el retorno a la educación superior ha estado más bien subiendo, particularmente entre 1990 y 2000 para luego mantenerse relativamente estable y muy superior al que se observa para las mujeres con educación media. En estas circunstancias no es extraño, entonces, que las brechas en la parte superior de la distribución de ingresos no muestren indicios de ceder. Eventualmente esa situación debería comenzar a revertirse en la medida que aumente con más fuerza la proporción de personas en la fuerza de trabajo con educación superior. La reducción en los retornos a las personas con educación secundaria parece estar íntimamente ligada a la ampliación de la oferta de personas con educación secundaria completa, que ha sido más mucho más rápida que en el caso de personas con educación superior.

En ese sentido debería constituir un motivo de preocupación que, tal como muestra el Cuadro $\mathrm{N}^{\circ} 6$, parece existir una fuerte desaceleración en el aumento de la cobertura de educación superior, particularmente a partir de 2003. Las razones de este fenómeno no son evidentes, pero es un problema que requiere atención. El aumento en la oferta de

${ }^{16}$ Para ver los canales a través de los cuales se puede materializar esta posibilidad puede revisarse Pavcnik y Goldberg (2007). 
personas con educación superior debería reducir el premio a este tipo de educación y contribuir a aplanar por arriba la distribución de ingresos, situación que hasta ahora no ha ocurrido y en el caso particular de las mujeres ha ido en la dirección contraria a la esperada.

CUADRO N 6: TASAS NETAS Y BRUTAS DE MATRÍCULA EN EDUCACIÓN SUPERIOR

\begin{tabular}{lcccccc}
\hline & 1990 & 1996 & 2000 & 2003 & 2006 & 2009 \\
\hline & & & & & & \\
Tasa neta & 12,8 & 21,0 & 22,1 & 26,0 & 27,4 & 29,1 \\
Tasa bruta & 15,6 & 26,1 & 30,9 & 37,8 & 38,3 & 39,8 \\
\hline
\end{tabular}

Fuente: Mideplan.

Con todo, como mostraba el Gráfico $\mathrm{N}^{\mathrm{0}} 1$, las generaciones más jóvenes muestran menores brechas educacionales. Esa situación en sí misma puede tener implicancias importantes sobre la desigualdad de los ingresos del trabajo. En efecto, si recordamos la ecuación (1) podemos desprender de ella, asumiendo que la escolaridad no está correlacionada con el error, la siguiente relación.

$$
\operatorname{Var}(\ln y)=\operatorname{Var}(\beta) \cdot S^{2}+\beta^{2} \cdot \operatorname{Var}(S)+\operatorname{cov}(\beta, S)+\operatorname{Var}(\mu)
$$

En el modelo tradicional $\beta$ toma exactamente el mismo valor para todos los niveles educativos. En ese sentido la varianza de $\beta$ sería igual a cero al igual que la covarianza entre $\beta$ y la escolaridad $\mathrm{S}$. La ecuación, entonces, se puede simplificar y expresar del siguiente modo:

$$
\operatorname{Var}(\ln y)=\beta^{2} \cdot \operatorname{Var}(S)+\operatorname{Var}(\mu)
$$

Esta ecuación es una primera aproximación, por cierto simplificada, para evaluar el potencial impacto de la reducción de las brechas de escolaridad en la desigualdad de ingresos que está ocurriendo en las nuevas generaciones. En efecto, aplicándolo para distintos grupos de edad se puede apreciar el efecto de la reducción en la varianza de la escolaridad sobre la variación de los ingresos del trabajo ${ }^{17}$. El Cuadro

\footnotetext{
${ }^{17}$ Una aplicación de este enfoque se encuentra en Lam y Schoeni (1993).
} 
No 7 exhibe tanto para 1990 como para 2009 los valores obtenidos para las distintas variables de la ecuación (3), es decir presenta una descomposición de la varianza de los ingresos.

El Cuadro No 7 ilustra las complejidades involucradas en la evolución de la distribución de ingresos. Al interior de cada grupo de edad se observa un aumento en la varianza de los ingresos, particularmente en los grupos más jóvenes. Ese aumento habría sido mayor si no hubiese ocurrido al mismo tiempo una disminución en la varianza de la escolaridad que compensó parte del incremento en la varianza de los errores $(\operatorname{Var}(\mu))$ atribuido a variables no observables. Si ésta no se hubiese modificado, la varianza de los ingresos para cada grupo de edad hubiese disminuido, porque los retornos a la escolaridad (expresados indirectamente por $\beta^{2}$ ) sólo sufrieron variaciones leves. Asimismo, es evidente a partir de esta evidencia que no se puede concluir que la menor varianza que se observa entre los más jóvenes se mantendrá con el paso del tiempo. Por una parte, la varianza de los ingresos no explicada por la educación aumenta $\mathrm{y}$, por otra, el retorno de la educación se eleva con la edad. Con todo, sólo por la disminución en las brechas de escolaridad la varianza de los ingresos para cada grupo de edad podría reducirse en un 20 por ciento.

Antes habíamos dicho (Cuadro $N^{\circ} 5$ ) que las brechas en los ingresos del trabajo se habían reducido en forma relevante, sobre todo

CUADRO N $^{\circ}$ 7: DESCOMPOSICIÓN DE LA VARIANZA DEL LOGARITMO NATURAL DE LOS INGRESOS DEL TRABAJO

\begin{tabular}{|c|c|c|c|c|c|c|c|c|}
\hline & 1990 & & & & 2009 & & & \\
\hline Edad & Var(lny) & $\beta^{2}$ & $\operatorname{Var}(\mathrm{S})$ & $\operatorname{Var}(\mu)$ & Var(lny) & $\beta^{2}$ & $\operatorname{Var}(\mathrm{S})$ & $\operatorname{Var}(\mu)$ \\
\hline $20-24$ & 0,509 & 0,00436 & 12,54 & 0,454 & 0,743 & 0,00406 & 8,23 & 0,702 \\
\hline $25-29$ & 0,663 & 0,00941 & 14,70 & 0,525 & 0,808 & 0,01061 & 12,37 & 0,677 \\
\hline $30-34$ & 0,856 & 0,01464 & 16,58 & 0,613 & 0,894 & 0,01254 & 14,00 & 0,718 \\
\hline $35-39$ & 0,894 & 0,01323 & 17,82 & 0,658 & 0,944 & 0,01538 & 14,59 & 0,720 \\
\hline $40-44$ & 0,887 & 0,01082 & 21,77 & 0,651 & 1,008 & 0,01232 & 15,53 & 0,817 \\
\hline $45-49$ & 0,933 & 0,01166 & 22,21 & 0,674 & 0,995 & 0,01232 & 16,21 & 0,795 \\
\hline $50-54$ & 1,045 & 0,01145 & 23,87 & 0,772 & 1,057 & 0,01299 & 17,36 & 0,831 \\
\hline $55-59$ & 1,186 & 0,01416 & 21,70 & 0,879 & 1,147 & 0,01103 & 21,31 & 0,912 \\
\hline $60-64$ & 1,219 & 0,01254 & 21,92 & 0,944 & 1,417 & 0,01742 & 25,01 & 0,981 \\
\hline
\end{tabular}

Fuente: Elaboración propia a partir de encuestas Casen 1990 y 2009. 
en la parte inferior de la distribución del ingreso. Ello no debería sorprender, porque no necesariamente ambos indicadores se mueven en la misma dirección. Para ver esto piénsese en los Gráficos N ${ }^{\text {os. }} 5$ y 6 . En ellos se veía que la tendencia entre 1990 y 2009 era de un aplanamiento de los retornos a la educación (y, por consiguiente, de las diferencias de ingresos) hasta los 12 años de escolaridad. Sin embargo, ello no se observaba para niveles de escolaridad mayores e incluso entre las mujeres se observaba un incremento en los retornos para esos niveles. Es perfectamente posible que en esas circunstancias aumente la varianza y exista un cierre en las brechas. Hay que recordar que la varianza es una medida de dispersión respecto de la media. El Cuadro $\mathrm{N}^{\mathrm{o}} 8$ ilustra esta situación al comparar los cambios en la brecha de los asalariados entre 1990 y 2009 y la varianza en los salarios por hora (más bien el logaritmo natural de estos).

Se aprecia que en 1996 y 2006 ambos indicadores van en la misma dirección. En el primer año aumentan las brechas salariales y aumenta la varianza de los ingresos. En 2006, en cambio, se reducen esas brechas y el segundo indicador también se reduce. En cambio en 2009 la brecha salarial es inferior a 1990, pero aun así la varianza de los salarios se incrementa. Este hecho se explica en una proporción importante, como sugiere el Cuadro $\mathrm{N}^{\circ}$ 9, por un aumento en la varianza de los salarios de las mujeres.

Ese aumento en la varianza de los salarios de las mujeres no se puede desligar del aumento en su tasa de ocupación. Ésta estaba por debajo del 30 por ciento en 1990 mientras que hacia fines de esta década esa tasa alcanzó casi un 40 por ciento. Además, a diferencia de lo que

CUADRO No 8: INDICADORES DE DESIGUALDAD PARA ASALARIADOS

\begin{tabular}{lccccc}
\hline & 1990 & 1996 & 2000 & 2006 & 2009 \\
\hline $90-10$ & 1,85 & 2,01 & 1,91 & 1,70 & 1,77 \\
$90-50$ & 1,20 & 1,18 & 1,25 & 1,13 & 1,21 \\
$50-10$ & 0,65 & 0,83 & 0,66 & 0,57 & 0,56 \\
Var (lny) & 0,66 & 0,76 & 0,64 & 0,57 & 0,74 \\
\hline
\end{tabular}

Fuente: Elaboración propia sobre la base de encuestas Casen. 
CUADRO No 9: VARIANZA DE LOS SALARIOS POR HORA (LOGARITMO NATURAL) DE HOMBRES Y MUJERES

\begin{tabular}{lcc}
\hline & 1990 & 2009 \\
\hline Hombres & 0,56 & 0,70 \\
Mujeres & 0,70 & 0,77 \\
Total & 0,66 & 0,74 \\
\hline
\end{tabular}

Fuente: Elaboración propia sobre la base de encuestas Casen.

ocurre con los hombres, su participación en la fuerza de trabajo difiere marcadamente para cada nivel de educación alcanzado. Esta irregular participación unida a la particular evolución que ha tenido el empleo para distintos grupos ayuda a entender el hecho que Chile no haya avanzado en reducir a un mayor ritmo su elevada desigualdad. Sobre ello trata la siguiente sección.

\section{La situación de empleo}

La evolución del empleo no ha sido particularmente positiva para la desigualdad de ingresos en nuestro país. Si bien, entre 1990 y 2009 se observa una tendencia al aumento en la tasa de empleo, neutralizada obviamente en aquellos años donde se observan desaceleraciones o crisis económicas profundas, hay grupos específicos que tienen dificultades para mantenerse vinculados al mundo del trabajo. El Gráfico N 7 muestra el comportamiento de la tasa de ocupación por quintil de ingresos y para el total de la población. Es claro que la tasa de ocupación total muestra una tendencia al alza, aunque como se advertía se redujo en 2000 y 2009, particularmente en esta última medición, como consecuencia de las situaciones económicas particulares que se vivieron esos años.

En el primer caso consecuencia de la crisis asiática de la que el país aún no se recuperaba del todo. En el segundo, producto de la profunda crisis económica que afectó a las economías industrializadas en la última parte de 2008 y gran parte de 2009. Lógicamente, Chile no se pudo sustraer del todo de ese fenómeno y el empleo se vio severamente 
GRÁFICO No 7: TASAS DE OCUPACIÓN POR QUINTIL DE INGRESOS ENTRE 1990 Y 2006

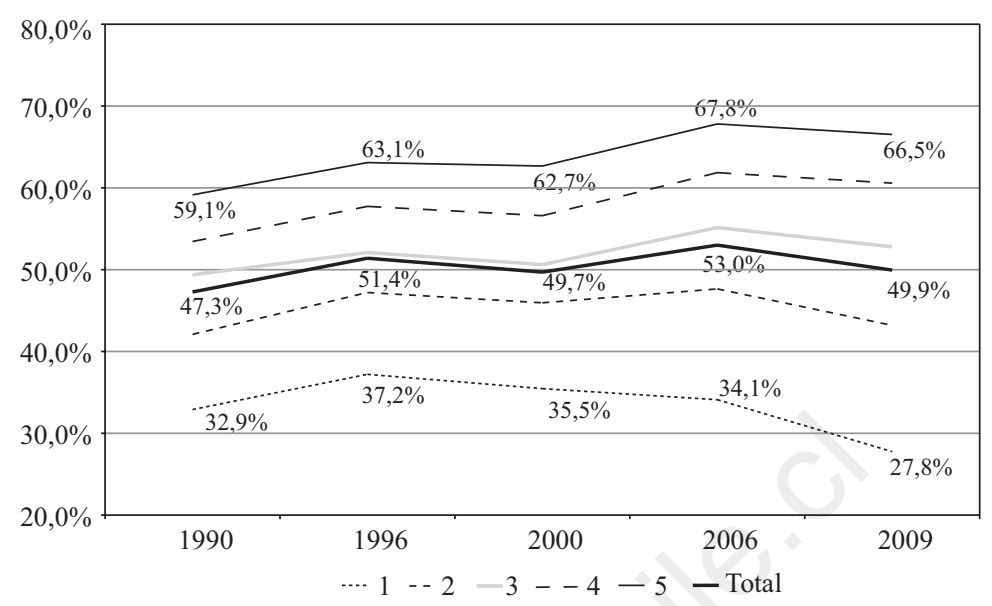

afectado. Pero ello no debe sorprender, dado el grado de integración de la economía nacional al resto del mundo. Se aprecia también que el grupo que más sufrió el embate de la crisis en términos de empleo fue el quintil de menores ingresos. Ahí, la tasa de empleo cayó en un 18,5 por ciento mientras que en el quinto quintil, el de mayores ingresos, esa tasa cayó sólo en un dos por ciento. Esa asimetría indudablemente es un golpe inmediato a la evolución de la desigualdad. Por cierto, hay en estos datos un sesgo de selección. En efecto, si hay una baja tasa de empleo en un hogar, la probabilidad de que quede en el primer quintil aumenta, porque los ingresos del trabajo son el componente más relevante de los ingresos autónomos. Aun teniendo presente este hecho no deja de sorprender la magnitud del ajuste en oportunidades ocupacionales. Y más allá de la crisis preocupa que el deterioro en sus oportunidades de empleo parece provenir desde antes, sugiriendo que hay un grupo de hogares que, a pesar de la tendencia general al aumento en las tasas de empleo, se está alejando del mercado del trabajo. En efecto, para el primer quintil de ingreso se observa un deterioro en la tasa de ocupación desde 1996 y el segundo quintil ha tenido dificultades para mantener el ritmo de crecimiento de la ocupación que se observa en el resto de los sectores. En un país con altas desigualdades, donde el empleo es un 
instrumento poderoso para cerrar brechas de ingreso, esa constatación es preocupante.

En el análisis de la evolución del empleo debe tratar de distinguirse entre fenómenos permanentes y transitorios, pero lamentablemente encuestas como la Casen miden un momento en el tiempo. En ese sentido, no se puede descartar que el fenómeno registrado sea uno en el cual las personas entran y salen con más frecuencia del empleo, es decir donde hay más rotación laboral. La evidencia proveniente de datos administrativos como los del Seguro de Cesantía sugiere que los contratos indefinidos se usan con menos frecuencia que en el pasado siendo reemplazados por un uso más frecuente de contratos a plazo fijo y por obra y faena. Así, podría ser el caso que las posibilidades efectivas de empleo hubiesen aumentado, pero ello no es registrado por una medición puntual en el tiempo. Sin embargo, no es evidente que una mayor rotación se traduzca en menores tasas de empleo en un momento en el tiempo. Además, en una circunstancia de estas características debería esperarse al menos un aumento de la participación en la fuerza de trabajo. Sin embargo, esa participación cae en el primer quintil de ingresos sugiriendo no sólo pérdida de empleo sin una deserción del mundo del trabajo.

Un análisis más detallado insinúa que detrás de la caída en la tasa de empleo del primer quintil de ingresos hay un retroceso en las oportunidades de empleo de los grupos menos calificados. El Cuadro $\mathrm{N}^{\mathrm{o}} 10$, por ejemplo, presenta la evolución en la tasa de empleo por grupos de edad y es claro que han disminuido las oportunidades de empleo de los más jóvenes quienes son típicamente las personas con menos califica-

CUADRO No 10: EVOLUCIÓN EN LAS TASAS DE EMPLEO POR EDADES (\%)

\begin{tabular}{llllll}
\hline Edad & 1990 & 1996 & 2000 & 2006 & 2009 \\
\hline $15-24$ & 32,6 & 32,7 & 26,5 & 29,9 & 27,4 \\
$25-29$ & 61,5 & 67,0 & 63,7 & 68,8 & 65,0 \\
$30-39$ & 64,5 & 67,6 & 67,4 & 71,9 & 71,6 \\
$40-49$ & 63,3 & 68,9 & 68,1 & 72,2 & 69,7 \\
$50-59$ & 51,2 & 58,4 & 59,3 & 64,9 & 62,9 \\
$60-69$ & 30,2 & 34,7 & 33,8 & 39,6 & 37,9 \\
\hline
\end{tabular}

Fuente: Elaboración propia a partir de encuestas Casen. 
ción. Los otros grupos, más allá de las fluctuaciones asociadas a los ciclos económicos, muestran una tendencia al alza en materia de empleo.

El mismo fenómeno, menores oportunidades de empleo para los menos calificados, se observa en el Cuadro $N^{0} 11$ que presenta la evolución en la tasa de ocupación por escolaridad.

Es claro que el grupo de menor escolaridad presenta una muy baja tasa de empleo y que ésta ha venido reduciéndose en el tiempo. En el grupo de 8 a 11 años si bien no se observa una tendencia declinante, se constata que su tasa de ocupación es reducida. En efecto, mientras en 2009 la tasa de empleo para el promedio de los países de la OCDE era 64,8 por ciento $^{18}$ (este número excluye a Chile, que a partir del año 2010 forma parte de esa organización), para nuestro país esa tasa alcanzaba el 55,7 por ciento en 2009 de acuerdo a la encuesta Casen de ese año, es decir era nueve puntos porcentuales más baja. Para igualar esa tasa, Chile tendría que haber tenido empleadas, en ese entonces, un poco más de un millón de personas adicionales para equiparar la tasa promedio de empleo de la OCDE. Debe reconocerse que dicha tasa promedio esconde importantes diferencias entre países existiendo varios que están por sobre 70 por ciento. Al mismo tiempo tasas como la nuestra son poco frecuentes: sólo cinco entre 30 países tienen una tasa de empleo inferior a 60 por ciento. De ellos tres están muy cerca del 60 por ciento. Así, no cabe duda que la tasa de ocupación nacional es modesta, reduciendo las posibilidades de avanzar en igualdad. Un fenómeno interesante es lo que ha sucedido con la tasa de ocupación de los hombres. El Gráfico No 8 muestra cómo esa tasa ha ido evolucionando para los hombres.

CUADRO No 11: $\quad$ EVOLUCIÓN EN LAS TASAS DE OCUPACIÓN POR ESCOLARIDAD (\%)

\begin{tabular}{lccccc}
\hline Años de educación & 1990 & 1996 & 2000 & 2006 & 2009 \\
\hline & & & & & \\
0 a 7 & 41,7 & 43,1 & 39,1 & 39,4 & 34,6 \\
8 a 11 & 42,3 & 46,2 & 43,8 & 45,8 & 42,4 \\
12 y más & 58,0 & 61,8 & 60,2 & 64,0 & 60,2 \\
\hline
\end{tabular}

Fuente: Elaboración propia a partir de encuestas Casen.

${ }^{18}$ Véase OCDE (2010). 
GRÁFICO No 8: EVOLUCIÓN DE LA TASA DE EMPLEO PARA HOMBRES MAYORES DE 15 AÑOS

(Promedios anuales)

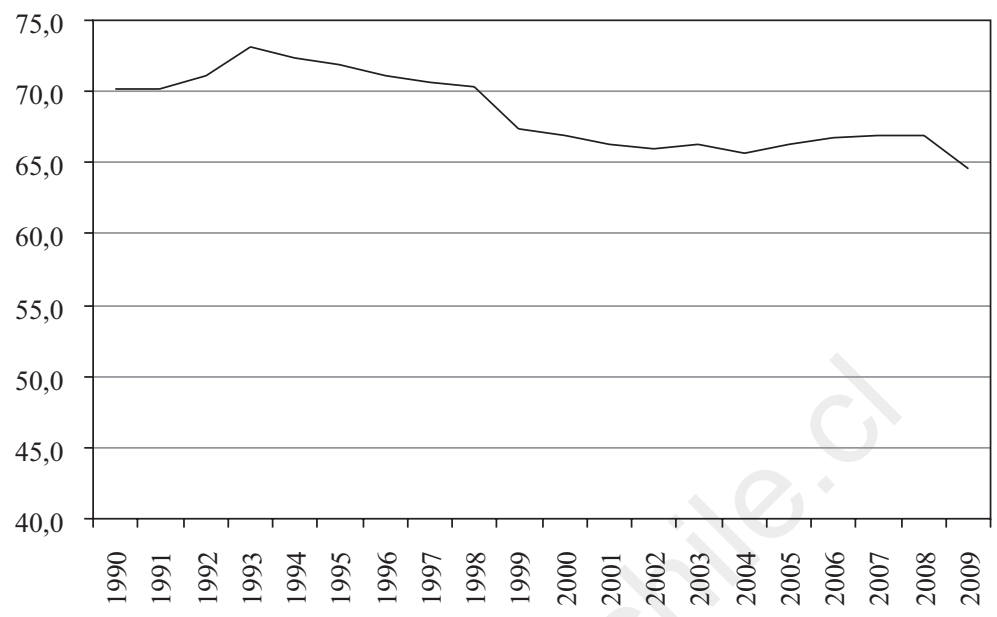

Fuente: Elaboración propia a partir de Encuesta de Empleo, INE.

Es evidente que ha habido una reducción importante en la tasa de ocupación de los hombres mayores de 15 años a partir de 1993, año en que alcanzó un 73,1 por ciento. Luego ésta ha descendido para alcanzar un mínimo de 65,7 por ciento en 2004. Luego se recuperó muy modestamente para alcanzar un 66,9 por ciento en 2007 y 2008. En 2009 volvió a declinar, aunque ese año la profunda crisis que vivió la economía internacional jugó un papel no despreciable. Interesantemente, este fenómeno va acompañado de un aumento en la tasa de ocupación de la mujer. En el mismo período, esto es entre 1993 y 2009, la tasa de empleo de la mujer de acuerdo al INE, subió de 31 a 37 por ciento. Una tendencia similar, aunque con tasas algo distintas, muestran las encuestas Casen. Las tasas de ocupación reportadas para las mujeres son aún muy bajas y es probable que su participación esté sesgada a favor de las mujeres de mayores habilidades y calificaciones. Por ello, no se puede descartar que su incorporación a la fuerza de trabajo haya sustituido en algunas áreas a hombres poco calificados. Esa sustitución puede tener diversos orígenes, pero es compatible con la idea de que las regulaciones disponibles o el progreso tecnológico en Chile no son 
particularmente amistosos con el trabajo poco calificado ${ }^{19}$. El hecho que el empleo en los sectores poco calificados muestre una tendencia declinante representa una luz de alerta sobre la disminución de brechas salariales que se reportaban para la parte inferior de la distribución de ingresos del trabajo. En efecto, si ese fenómeno es consecuencia de una "expulsión" desde la fuerza de trabajo de las personas menos calificadas, la compresión salarial pierde atractivo. En todo caso, debe reconocerse que ese hecho no ha afectado el mayor aplanamiento que se ha observado en la distribución de los ingresos autónomos de los hogares, de nuevo en la parte inferior de la distribución. Ello sugiere que la salida de esos trabajadores del mercado laboral no ha afectado negativamente, en promedio, la capacidad de generación de ingresos de esos hogares. Por supuesto, muchos hogares podrían estar mejor si esas personas estuviesen en un puesto de trabajo. Además, el gradual avance hacia una menor desigualdad de los ingresos autónomos per cápita se habría reforzado.

\section{Conclusiones}

En el contexto de elevada desigualdad en el que se desarrolla Chile, resulta interesante realizar el ejercicio de evaluar si los indicadores habituales de desigualdad, que van a seguir siendo relevantes en el análisis mientras no exista mejor información sobre movilidad social y sobre los cambios que afectan a los mismos hogares a través del tiempo, presentan evidencia respecto de la dirección en la que podrían moverse estos indicadores en los próximos lustros. El análisis preliminar que se presenta en este estudio sugiere que hay buenas noticias. A pesar de la crisis económica de los años 2008 y 2009, que afectaron los indicadores de desigualdad, subyacen en los datos cambios que permiten pensar que en el futuro se podrían ir sumando avances en igualdad. Ellos tienen que ver con la reducción en las brechas de escolaridad, las menores diferencias salariales en la parte inferior de la distribución y el aumento más rápido que se observa en la última década en el ingreso autónomo per cápita de los hogares de menores ingresos.

${ }^{19}$ Sobre esto, véase Beyer y Sapelli (2009). 
Al mismo tiempo se observa que el aumento en la escolaridad ha sido crucial para reducir las diferencias salariales entre las personas que tienen educación media y menos. Ello ha ocurrido como resultado de una caída relativa en el retorno a la educación secundaria, que a su vez es consecuencia del importante aumento en la oferta de graduados de educación secundaria. En cambio, el retorno a la educación superior se ha mantenido en niveles elevados e incluso en el caso de las mujeres se ha incrementado. Ello obedece principalmente al hecho que el aumento en la oferta de graduados con educación superior incompleta o completa ha sido pequeño respecto de la fuerza de trabajo. En efecto, entre 1990 y 2009 pasó de 22 a 28 por ciento de la fuerza de trabajo. En cambio, la proporción de personas con 9 a 12 años de escolaridad aumentó en diez puntos porcentuales de 38 a 48 por ciento; los miembros de la fuerza de trabajo con educación secundaria completa, en particular, pasaron de representar un 21 a un 35 por ciento del total entre 1990 y 2009.

En los próximos años cabría esperar que se observe un aumento en la oferta relativa de graduados con educación superior que coloque presión a los retornos de esta educación, contribuyendo a aplanar los ingresos asociados a este tipo de educación. Ello debería ser un aporte a la reducción de la desigualdad de ingresos a través de su impacto en las diferencias salariales que deberían disminuir. Para consolidar esta tendencia probablemente deba ponerse más atención al relativo estancamiento que se observa en la cobertura de educación superior de acuerdo a la información que se desprende de las últimas encuestas Casen.

El talón de Aquiles de la política redistributiva es el empleo. Hay bajas tasas promedio de ocupación en Chile, pero además mucha heterogeneidad, al grado que estas tasas en el quintil de más elevados ingresos es de 67 por ciento y en el quintil de más bajos ingresos es de sólo un 28 por ciento. Pero, además de estas enormes diferencias, se observa una preocupante declinación en la participación laboral de los grupos menos calificados. Una política redistributiva no puede renunciar a proveer de empleos a la población menos calificada, toda vez que no hay un instrumento más efectivo en plazos razonables para avanzar en equidad. 


\section{REFERENCIAS BIBLIOGRÁFICAS}

Arellano, José Pablo (2004). "Políticas Sociales para el Crecimiento con Equidad: Chile 1990-2002”. Cieplan, Serie Estudios Socio/Económicos N²6, abril.

Beyer, Harald (1999). "Educación y Desigualdad de Ingresos: Una Nueva Mirada". Centro de Estudios Públicos, Serie Documentos de Trabajo, No 297, agosto.

(2005). "Productividad, Desigualdad y Capital Humano: Los Complejos Desafíos de Chile”. Estudios Públicos, 97 (verano), pp. 59-98.

Beyer, Harald y Carmen Le Foulon (2002). "Un Recorrido por las Desigualdades Salariales en Chile”. Estudios Públicos, 85 (verano), pp. 139-175.

Beyer, Harald y Claudio Sapelli (2009). "Hacia una Política Social Efectiva y Eficiente". En Harald Beyer, Alfredo Joignant y Cristóbal Marín (compiladores), El Chile que Viene: De Dónde Venimos, Dónde Estamos y a Dónde Vamos. Santiago: Universidad Diego Portales y Centro de Estudios Públicos.

Blau, Francine y Lawrence Kahn (1996). "International Differences in Male Wage Inequality". (With Lawrence M. Kahn) Journal of Political Economy, agosto. (2005). "Do Cognitive Test Scores Explain Higher US Wage Inequality?" Review of Economics and Statistics, febrero.

Kenworthy, Lane (2008). Jobs with Equality. Oxford University Press.

Lam, David y Robert Schoeni (1993). "Effects of Family Background on Earnings and Returns to Schooling: Evidence from Brazil". Journal of Political Economy, Vol. $101 \mathrm{~N}^{\circ} 4$ (agosto), pp. 213-243.

Larrañaga, Osvaldo y Rodrigo Herrera (2008). "Los Recientes Cambios en la Desigualdad y la Pobreza en Chile”. Estudios Públicos, 109 (verano), pp. 149186.

Mincer, Jacob (1974). Schooling, Experience and Earnings. Nueva York: National Bureau of Economic Research.

OCDE (2010). Employment Outlook 2010. París.

Pavcnik, Nina y Penny Goldberg (2007). "Distributional Effects of Globalization in Developing Countries". Journal of Economic Literature, Vol. 45 № 1 (marzo), pp. 39-82.

Sapelli, Claudio (2009). "The Evolution of the Intergenerational Mobility of Education in Chile by Cohorts: Facts and Possible Causes". Instituto de Economía, Universidad Católica, № 348 .

Williamson, Jeffrey (2009). "Five Centuries of Latin American Inequality". Mimeo, Universidad de Harvard. 